\title{
Death due to intravenous use of $\alpha$-pyrrolidinopentiophenone
}

\begin{abstract}
Intoxication with synthetic cathinones (psychoactive designer drugs) can involve cardiovascular, autonomic, neuromuscular and neuropsychiatric features. We report a case of cardiac arrest and subsequent death in a 44-year-old man after intravenous use of one such drug $-\alpha$-pyrrolidinopentiophenone. We believe this is the first death associated with this drug to be reported in Australia. Currently, no specific antidote exists for cathinone exposure.
\end{abstract}

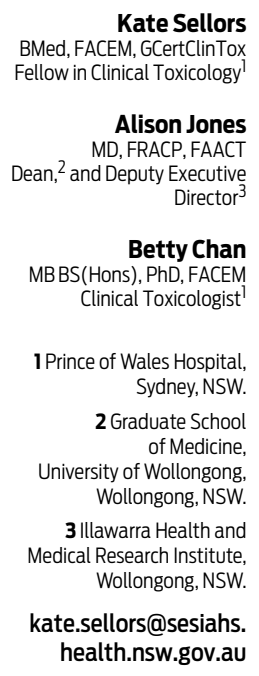

doi: 10.5694/mjal3.00203

\section{Clinical record}

A 44-year-old man with a history of substance misuse injected himself with a powder named "Smokin' Slurry Scrubba" that he and his girlfriend had purchased over the counter from a shop. Soon after injecting the powder, the man stripped off all his clothes, jumped over a barbed-wire fence and smashed a window. He was restrained and pinned to the floor by security staff while emergency services were called, during which time he suffered a cardiac arrest.

Cardiopulmonary resuscitation (CPR) was commenced by bystanders. On arrival of ambulance paramedics, the patient was confirmed to be in asystolic cardiac arrest and the paramedics continued CPR. Spontaneous circulation was restored after a further 3 minutes of advanced cardiac life support protocol. He was intubated at the scene by the paramedics. The total duration of cardiac arrest was estimated to be 16 minutes. His vital signs immediately after the arrest were: Glasgow Coma Scale (GCS) score, 3; blood pressure, $65 / 20 \mathrm{mmHg}$; heart rate, 85 beats/min; tympanic temperature, $39.7^{\circ} \mathrm{C}$. An adrenaline infusion was started at $30 \mu \mathrm{g} / \mathrm{min}$ to treat persistent systemic hypotension.

On arrival at the emergency department, about 1 hour after the cardiac arrest, the patient's temperature had climbed to $39.9^{\circ} \mathrm{C}$ and he was still hypotensive (blood pressure, $83 / 45 \mathrm{mmHg}$ ). On physical examination, he had a GCS score of 3, and his pupils were dilated (right, $5 \mathrm{~mm}$; left, $6 \mathrm{~mm}$ ) and unreactive to light. A venous blood gas and serum analysis using samples taken on arrival at the emergency department showed a severe metabolic and respiratory acidosis with evidence of hyperkalaemia, rhabdomyolysis, ischaemic hepatitis, elevated lactate level and acute renal failure (Box 1). An electrocardiogram showed a sinus tachycardia of 126 beats/min with normal intervals and no ischaemic changes, and results of a non-contrast computed tomography scan of his brain were reported as normal. A total of 5 litres of cooled crystalloid solution was given intravenously. Within an hour of arrival at the emergency department, the patient's temperature had normalised to $37.2^{\circ} \mathrm{C}$ and his blood pressure had stabilised while on the adrenaline infusion. Other initial treatment included intravenous calcium gluconate and sodium bicarbonate for treatment of hyperkalaemia. He began to respond to painful stimuli and required sedation.

On arrival at the intensive care unit, about 6 hours after the cardiac arrest, the patient's pupils had become equal and reactive at $2 \mathrm{~mm}$ diameter. Repeated blood analysis revealed abatement of his lactic acidosis, but worsening of his rhabdomyolysis (Box 1). An echocardiogram taken at this time was normal. Results of a qualitative urine drug screen (Roche Diagnostics immunoassay) using a sample taken on the patient's arrival at the intensive care unit were positive for benzodiazepines but negative for amphetamines, cannabis, cocaine and opioids. There was also evidence of disseminated intravascular coagulation - an international normalised ratio of 2.5 (reference interval [RI], 0.8-1.1), an activated partial thromboplastin time of 82.2s (RI, 25.0-37.0 s) and a plasma fibrinogen level of $1.4 \mathrm{~g} / \mathrm{L}(\mathrm{RI}, 2.20-4.30 \mathrm{~g} / \mathrm{L})$ - but no clinical evidence of bleeding. Due to the increasing creatine kinase levels, the patient was started on continuous venovenous haemodiafiltration in an attempt to mitigate further renal damage.

About 24 hours after the cardiac arrest, the patient developed signs of raised intracranial pressure with fixed dilated pupils and haemodynamic instability; he did not respond to noxious stimuli and he had lost brain stem reflexes. A non-contrast computed tomography scan of his brain showed diffuse cerebral oedema with tonsillar herniation and multiple areas of cerebral infarction (Box 2). About 43 hours after the cardiac arrest, the patient was declared brain dead by clinical criteria, and supportive care was withdrawn.

The only physical findings of note on postmortem examination were cerebellar tonsillar herniation and pulmonary oedema. There was no evidence of trauma. Coronial analysis of antemortem and postmortem blood samples (using liquid chromatography mass spectrophotometry) revealed the qualitative presence of $\alpha$-pyrrolidinopentiophenone ( $\alpha$-PVP). No other common drugs of misuse were detected. 
1 Results of a venous blood gas analysis for a 44-year-old man after he had a cardiac arrest due to $\alpha$-pyrrolidinopentiophenone toxicity

\begin{tabular}{lccc} 
Component & $\begin{array}{c}\text { hh after } \\
\text { arrest }\end{array}$ & $\begin{array}{c}6 \text { h after } \\
\text { arrest }\end{array}$ & $\begin{array}{c}\text { Reference } \\
\text { interval }\end{array}$ \\
\hline $\mathrm{pH}$ & 6.62 & 7.21 & $7.35-7.43$ \\
Partial pressure of carbon dioxide $(\mathrm{mmHg})$ & 90 & 42 & $32-45$ \\
Bicarbonate (mmol/L) & 8.8 & 16.1 & $22-32$ \\
Lactate (mmol/L) & 29.0 & 4.9 & $<2.0$ \\
Potassium (mmol/L) & 6.2 & 3.3 & $3.6-5.1$ \\
Urea (mmol/L) & 13.4 & 12.4 & $2.9-7.1$ \\
Creatinine (jmol/L) & 201 & 169 & $60-110$ \\
Alanine transaminase (U/L) & 1976 & 1913 & $<45$ \\
Aspartate transaminase (U/L) & 1818 & 2457 & $<45$ \\
Creatine kinase (U/L) & 2763 & 24660 & $<200$ \\
Myoglobin ( $\mathrm{mg} / \mathrm{L})$ & - & 43190 & $10-92$
\end{tabular}

2 Non-contrast computed tomography scans of the brain of a 44-year-old man after he had a cardiac arrest due to $\alpha$-pyrrolidinopentiophenone toxicity

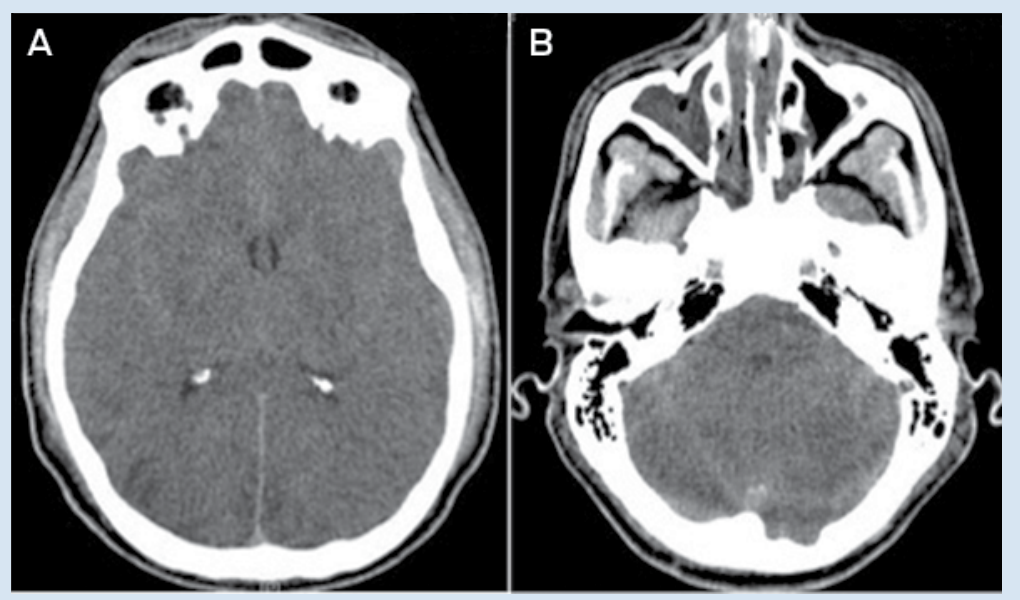

Scans taken 24 hours after cardiac arrest showing diffuse cerebral oedema with tonsillar herniation and multiple areas of cerebral infarction ( $\boldsymbol{A}$, level of the basal ganglia; $\boldsymbol{B}$, level of the mid posterior fossa).

3 Clinical features of synthetic cathinone intoxication

System

Cardiovascular

Autonomic

Neuromuscular

Neuropsychiatric

\section{Effects}

Tachycardia, vasoconstriction, systemic hypertension, arrhythmias, cardiovascular collapse, myocardial infarction,

Sympathetic hyperstimulation (autonomic hyperarousal), mydriasis, hypertension, hyperthermia

Seizures, stroke, tremors, muscle spasm, cerebral oedema

Agitation, hallucinations, panic attacks, paranoia, anxiety, insomnia, anorexia, depression, suicidal ideation, violence (self-mutilation, suicide and homicidal activity)

The formal conclusion of a subsequent coronial inquest was that the patient had died from cardiac arrest and cerebral oedema, which were the results of $\alpha$-PVP toxicity. The possibility of physical restraint and positional asphyxia contributing to his death was raised but these factors were considered not to be the primary cause of death.

\section{Discussion}

To our knowledge, this is the first death due to $\alpha$-PVP use reported in Australia. Assessing all the evidence, our patient died from the expected complications of exposure to $\alpha$-PVP.

$\alpha-P V P$ is a synthetic cathinone designer drug that is misused for its stimulant and psychoactive effects. It is an analogue of pyrovalerones ${ }^{1}$ such as 3,4-methylenedioxypyrovalerone (MDPV). Synthetic cathinones such as $\alpha$-PVP are widely sold on the internet; they are commonly referred to as "bath salts", "legal highs" or "research chemicals", and often labelled "not for human consumption" in an effort to evade regulatory control. ${ }^{2}$ Synthetic cathinones are also structural analogues of cathinone, the naturally occurring $\beta$-ketone amphetamine analogue found in the Catha edulis (Khat) plant.

As structural analogues of $\beta$-ketone amphetamines, cathinones are expected to have amphetamine-like effects. Many cathinones have been shown to be inhibitors of monoamine transporters, but selectivity of cathinones for serotonin, noradrenaline and dopamine transporters varies considerably in vitro. ${ }^{3}$ Pyrovalerone-cathinones (eg, MDPV) are potent and selective dopamine and noradrenaline uptake blockers, but are not effective releasers of monoamines. The potency of these drugs on the noradrenaline and dopamine transporters is associated with their stimulant and psychoactive effects.

Synthetic cathinones are most commonly nasally insufflated (snorted) or ingested, but rectal insertion, inhalation, and intravenous and intramuscular injection have also been described. ${ }^{2,4}$ Desirable effects of cathinones reported by users include increases in energy, empathy and libido.

The effects of synthetic cathinones (as a group) are said to begin to occur within 15-45 minutes of exposure and the desired effects last from 2 to 7 hours. ${ }^{4}$ However, the undesirable effects can last from hours to days. Clinical features of synthetic cathinone intoxication include cardiovascular, autonomic, neuromuscular and neuropsychiatric symptoms and signs (Box 3). 2,4,5

No specific antidote exists for cathinone exposure, and there are limited published data on specific management strategies. Current practice is based on experience treating patients intoxicated with other sympathomimetic drugs, for which supportive care is the mainstay of treatment. ${ }^{4}$ Benzodiazepines are recommended for control of agitation and seizures, and for treatment of hypertension and tachycardia, and passive or active cooling is recommended for treatment for hyperthermia that is not resolved with anxiolysis and sedation. ${ }^{4,5}$ In Australia, poisons information centres can be contacted for further advice.

While there have been a few media reports of violence, aggression and deaths related to use of $\alpha$-PVP, few cases have been reported in peer-reviewed journals. One article describes the deaths of five patients for whom $\alpha$-PVP was detected in postmortem blood, but this does not necessarily mean that $\alpha$-PVP intoxication was the cause of death or that $\alpha$-PVP was the only drug present. ${ }^{6}$ Another article describes a case of parenteral MDPV overdose, confirmed by a quantitative assay of MDPV in the patient's blood, in which the clinical course was very similar to that of our patient. ${ }^{7}$

Our patient probably died as a consequence of the complications of $\alpha$-PVP exposure. The time of death and 
clinical course fit with the features of autonomic hyperarousal: psychomotor agitation, delirium, tachycardia and hyperthermia, and subsequent cardiac arrest, rhabdomyolysis, renal failure, hepatic injury, anoxic brain injury and death. These features have also been described as "excited delirium syndrome". ${ }^{8}$ Excited delirium syndrome as a diagnostic entity is controversial and widely debated in the medical literature. The role of excessive stimulation of the sympathetic nervous system in subsequent cardiac death after drug exposure has also been widely debated. It has been postulated that overstimulation of the heart by catecholamines leads to increased contractility, blood pressure and heart rate, resulting in increased oxygen demand, followed by myocardial ischaemia, fatal cardiac arrhythmias and sudden death.

The single biggest factor influencing death from $\alpha-P V P$ intoxication as the outcome in this case was the route of exposure to the drug - that is, intravenous injection. From toxicokinetic first principles, this results in faster and higher blood concentrations of drugs compared with ingestion and nasal insufflation as routes of exposure.

Synthetic cathinones have been present in notable quantities in Australia since the mid 2000s. While the exact prevalence of use in Australia is difficult to determine, the availability and popularity of synthetic cathinones has increased over the past decade, as evidenced by surveys of regular drug users, wastewater analyses and drug seizures by the Australian Federal Police. ${ }^{9}$

Our patient tested negative for amphetamines when a urine drug screen was performed. Testing for $\alpha$-PVP was only available through coronial investigation. This highlights that medical professionals in Australia need to be aware of the limitations of commonly available drug screens, which might not detect new psychoactive substances.

Competing interests: No relevant disclosures.

Received 10 Dec 2013, accepted 14 Mar 2014.

1 Sauer C, Peters FT, Haas C, et al. New designer drug alphapyrrolidinovalerophenone (PVP): studies on its metabolism and toxicological detection in rat urine using gas chromatographic/mass spectrometric techniques. J Mass Spectrom 2009; 44: 952-964.

2 Prosser JM, Nelson LS. The toxicology of bath salts: a review of synthetic cathinones. J Med Toxicol 2012; 8: 33-42.

3 Simmler LD, Buser TA, Donzelli M, et al. Pharmacological characterization of designer cathinones in vitro. Br J Pharmacol 2013; 168: 458-470.

4 Rosenbaum CD, Carreiro SP, Babu KM. Here today, gone tomorrow ... and back again? A review of herbal marijuana alternatives (K2, Spice), synthetic cathinones (bath salts), kratom, Salvia divinorum, methoxetamine, and piperazines. J Med Toxicol 2012; 8: 15-32.

5 Ross EA, Watson M, Goldberger B. "Bath salts" intoxication. NEngl J Med 2011; 365: 967-968.

6 Marinetti LJ, Antonides HM. Analysis of synthetic cathinones commonly found in bath salts in human performance and postmortem toxicology: method development, drug distribution and interpretation of results. J Anal Toxicol 2013; 37: 135-146.

7 Murray BL, Murphy CM, Beuhler MC. Death following recreational use of designer drug "bath salts" containing 3,4-methylenedioxypyrovalerone (MDPV). J Med Toxicol 2012; 8: 69-75.

8 Vilke GM, DeBard ML, Chan TC, et al. Excited delirium syndrome (ExDS): defining based on a review of the literature. J Emerg Med 2012; 43: 897-905.

9 Bright S. New and emerging drugs. Not for human consumption: new and emerging drugs in Australia. Prev Res [internet]. 2013; Apr: 1-16. http:// www.druginfo.adf.org.au/attachments/957_PreventionResearch Newandemergingdrugs_Aprl3.pdf (accessed Nov 2013). 